\title{
Influence of the Porosity of Starch-Based Fiber Mesh Scaffolds on the Proliferation and Osteogenic Differentiation of Bone Marrow Stromal Cells Cultured in a Flow Perfusion Bioreactor
}

\author{
MANUELA E. GOMES, Ph.D., ${ }^{1-3}$ HEIDI L. HOLTORF, Ph.D., ${ }^{3}$ \\ RUI L. REIS, Ph.D., ${ }^{1,2}$ and ANTONIOS G. MIKOS, Ph.D. ${ }^{3}$
}

\begin{abstract}
This study investigates the influence of the porosity of fiber mesh scaffolds obtained from a blend of starch and poly( $\varepsilon$-caprolactone) on the proliferation and osteogenic differentiation of marrow stromal cells cultured under static and flow perfusion conditions. For this purpose, biodegradable scaffolds were fabricated by a fiber bonding method into mesh structures with two different porosities-50 and $\mathbf{7 5 \%}$. These scaffolds were then seeded with marrow stromal cells harvested from Wistar rats and cultured in a flow perfusion bioreactor or in 6-well plates for up to 15 days. Scaffolds of $75 \%$ porosity demonstrated significantly enhanced cell proliferation under both static and flow perfusion culture conditions. The expression of alkaline phosphatase activity was higher in flow cultures, but only for cells cultured onto the higher porosity scaffolds. Calcium deposition patterns were similar for both scaffolds, showing a significant enhancement of calcium deposition on cellscaffold constructs cultured under flow perfusion, as compared to static cultures. Calcium deposition was higher in scaffolds of $\mathbf{7 5 \%}$ porosity, but this difference was not statistically significant. Observation by scanning electron microscopy showed the formation of pore-like structures within the extracellular matrix deposited on the higher porosity scaffolds. Fourier transformed infrared spectroscopy with attenuated total reflectance and thin-film X-ray diffraction analysis of the cell-scaffold constructs after $\mathbf{1 5}$ days of culture in a flow perfusion bioreactor revealed the presence of a mineralized matrix similar to bone. These findings indicate that starch-based scaffolds, in conjunction with fluid flow bioreactor culture, minimize diffusion constraints and provide mechanical stimulation to the marrow stromal cells, leading to enhancement of differentiation toward development of bone-like mineralized tissue. These results also demonstrate that the scaffold structure, namely, the porosity, influences the sequential development of osteoblastic cells and, in combination with the culture conditions, may affect the functionality of tissues formed in vitro.
\end{abstract}

\section{INTRODUCTION}

N BONE TISSUE ENGINEERING STRATEGIES based on cellscaffold constructs, the macro- and microstructural properties of the scaffolds, in conjunction with the cell culture environment, may play a very important role in the proliferation and differentiation of cells into the desired phenotype and, consequently, affect the performance of the construct after implantation. Bone tissue formation by osteogenic cells, such as marrow stromal

\footnotetext{
13Bs Research Group, Biomaterials, Biodegradables and Biomimetics, University of Minho, Campus de Gualtar, Braga, Portugal.

${ }^{2}$ Department of Polymer Engineering, University of Minho, Campus de Azurém, Guimarães, Portugal.

${ }^{3}$ Department of Bioengineering, Rice University, Houston, Texas.
} 
cells, is characterized by sequential events involving cell proliferation, expression of osteoblastic markers, and the synthesis, deposition, and mineralization of a collagenous matrix. ${ }^{1}$ These events are greatly affected not only by the type of scaffold material in which the cells are seeded, ${ }^{2-6}$ but also by the culture environment. . $^{3,4,7}$

Adequate porosity and surface area are widely recognized $^{8-11}$ as important parameters in the design of scaffolds for tissue engineering. Other architectural features, such as pore morphology and interconnectivity between pores of the scaffolding materials, are also suggested to be important for cell seeding, migration, growth, mass transport, gene expression, and new tissue formation in three dimensions. ${ }^{8,9,12}$ For example, fiber mesh scaffolds typically exhibit large surface areas for cell attachment and high pore interconnectivity for adequate diffusion of nutrients.

In traditional static cell culture systems, cells within scaffold constructs will receive nutrients only by diffusion from the surrounding media. ${ }^{3,7}$ Thus, with static culture, high cell densities on the scaffold exteriors often deplete nutrient supplies before these nutrients can diffuse to the scaffold interiors. ${ }^{3}$ These diffusional limitations in static culture become more pronounced with decreasing scaffold porosity. Cells in the interiors of statically cultured scaffolds may ultimately become necrotic due to a lack of nutrients and an excess of metabolic waste products. $^{3,11}$ In addition to these transport limitations, static culture systems also fail to provide any mechanical stimulation to cell-seeded scaffolds. It is well known that bone cells are sensitive to mechanical stimulation, ${ }^{13,14}$ a constant in their natural environment. The absence of mechanical stresses may therefore hinder cell development in vitro. ${ }^{13}$ These findings have motivated the development of enhanced culture systems, such as the flow perfusion bioreactor, ${ }^{3,15-17}$ which simultaneously provides sufficient transport of nutrients and wastes and continuous mechanical stimulation of cells, $3,17,18$ creating dynamic culture environments that support in vitro formation of 3-D bone-like tissue.

Previous studies ${ }^{19}$ demonstrated that biodegradable starch-based scaffolds were able to support attachment, proliferation, and differentiation of bone marrow stromal cells. The present study examines the influence of fiber mesh scaffold porosity on cell proliferation and differentiation. In particular, scaffolds were fabricated from a blend of starch and poly( $\varepsilon$-caprolactone), seeded with rat bone marrow stromal cells, and cultured statically or under flow perfusion to address the following experimental questions: 1) What is the role/influence of scaffold porosity in the sequential development of cell-scaffold constructs cultured under flow perfusion as compared to static culture? 2) Does the porosity of the scaffolds and/or culture conditions affect the structure and composition of the tissue formed in vitro?

\section{MATERIALS AND METHODS}

\section{Scaffold preparation and characterization}

Two different scaffolds based on SPCL (a 30:70 wt\% blend of starch with poly( $\varepsilon$-caprolactone) $)$ were prepared by a fiber-bonding process consisting of cutting and sintering melt-spun fibers with a diameter of approximately $180 \mu \mathrm{m}$. The different porosities of the fiber meshes were obtained using different amounts (by weight) of fibers. The porosity of the scaffolds was determined by microcomputerized tomography $(\mu \mathrm{CT})$ (ScanCo Medical $\mu$ CT 80, Bassersdorf, Switzerland) at a resolution of $10 \mu \mathrm{m}$ ( $n \geq 3$ per formulation). ${ }^{20}$ The morphology of the porous structure was further characterized using a scanning electron microscope (Leica Cambridge S360, Leica Cambridge, United Kingdom) after sputter coating the samples with gold (Jeol JFC 1100, Jeol, Peabody, MA).

All samples were cut into discs of approximately $8 \mathrm{~mm}$ diameter and 1.5-2 $\mathrm{mm}$ height and sterilized using ethylene oxide. Prior to cell seeding, the scaffolds were immersed in $30 \mathrm{~mL}$ of serum-free media in $50 \mathrm{~mL}$ tubes. Air was removed from the scaffold pores by generating vacuum with a $30 \mathrm{~mL}$ syringe equipped with an 18-gauge needle. The scaffolds were left in serum-free media overnight to allow swelling.

\section{Isolation and expansion of rat bone marrow stromal cells}

Rat bone marrow stromal cells were obtained from the femora and tibiae of 125-150 g male Wistar rats (Harlan, Indianapolis, IN). The isolation and culturing procedures of the rat bone marrow stromal cells were previously described. ${ }^{21}$ Briefly, femora and tibiae were removed and washed in medium with a 10-fold higher antibiotic concentration than present in normal culture medium, which is composed of minimum essential medium ( $\alpha$-MEM Eagle, Sigma, St. Louis, MO) supplemented with $10 \%$ fetal calf serum (FCS, Gemini, Calabasas, CA), $50 \mu \mathrm{g} / \mathrm{mL}$ gentamicin, $100 \mu \mathrm{g} / \mathrm{mL}$ ampicillin, and $0.3 \mu \mathrm{g} / \mathrm{mL}$ fungizone. The epiphyses were cut off, and the diaphyses were flushed with $5 \mathrm{~mL}$ of complete culture medium, the same that was used for the entire experimental period, consisting of minimum essential medium supplemented with $10 \%$ FCS, $50 \mu \mathrm{g} / \mathrm{mL}$ ascorbic acid (Sigma Chemical), $50 \mu \mathrm{g} / \mathrm{mL}$ gentamicin, $100 \mu \mathrm{g} / \mathrm{mL}$ ampicillin, $0.3 \mu \mathrm{g} / \mathrm{mL}$ fungizone, $10 \mathrm{mM} \beta$ glycerophosphate (Sigma), and $10^{-8} \mathrm{M}$ dexamethasone (Sigma). The bone marrow obtained from all the rats was pooled and plated in $75 \mathrm{~cm}^{2}$ flasks. The cells were cultured at $37^{\circ} \mathrm{C}$ in a humidified atmosphere with $5 \% \mathrm{CO}_{2}$. The culture medium was refreshed after $24 \mathrm{~h}$ and thereafter every 2 days until day 6 . 


\section{Cell seeding on starch-based scaffolds}

At near confluence, after 6 days of primary culture, the adherent cells were enzymatically lifted using trypsin/ EDTA $(0.25 \%$ w/v trypsin/0.02\% EDTA; Sigma), concentrated by centrifugation at $400 \mathrm{~g}$ for $5 \mathrm{~min}$ and re-suspended in complete medium. Subsequently, the scaffolds were inserted into cassettes (small containers into which the scaffolds are press-fitted and loaded into the bioreactor after cell seeding) that were then placed in 6-well plates. Each scaffold was then seeded with $300 \mu \mathrm{L}$ of a cell suspension containing $5 \times 10^{5}$ cells and incubated for $2 \mathrm{~h}$. Next, 10 $\mathrm{mL}$ of complete medium was added to each well and the seeded scaffolds were further incubated overnight to allow for cell attachment. The following day seeded scaffolds were placed into fresh 6-well plates for static culture or into the flow perfusion bioreactor and cultured in complete media for 7 and 15 days ( $n=6$ scaffolds for each scaffold formulation at each time point for each culture condition).

\section{Cell culture: the flow perfusion culture system}

The bioreactor used in this study has been described in detail elsewhere. ${ }^{15,16}$ Briefly, this bioreactor consists of 6 flow chambers, each one containing a cassette into which the scaffold is press-fit. Gas-permeable silicon tubing connects each flow chamber with a peristaltic pump and a medium reservoir. Each chamber has its own independent pumping circuit, but all pumps draw media from a common reservoir. For these experiments, culture medium was pumped continuously at a flow rate of 1 $\mathrm{mL} / \mathrm{min}$ from the supply reservoir, through the cell-scaffold constructs, and back to the reservoir. The total volume of medium in the flow system was $210 \mathrm{~mL}$. In the static culture, $10 \mathrm{~mL}$ was added to each scaffold. In both culture systems, media were changed every 3 days. The entire flow perfusion bioreactor was maintained in an environment of $37^{\circ} \mathrm{C}$ with $5 \% \mathrm{CO}_{2}$. Figure 1 presents a flow

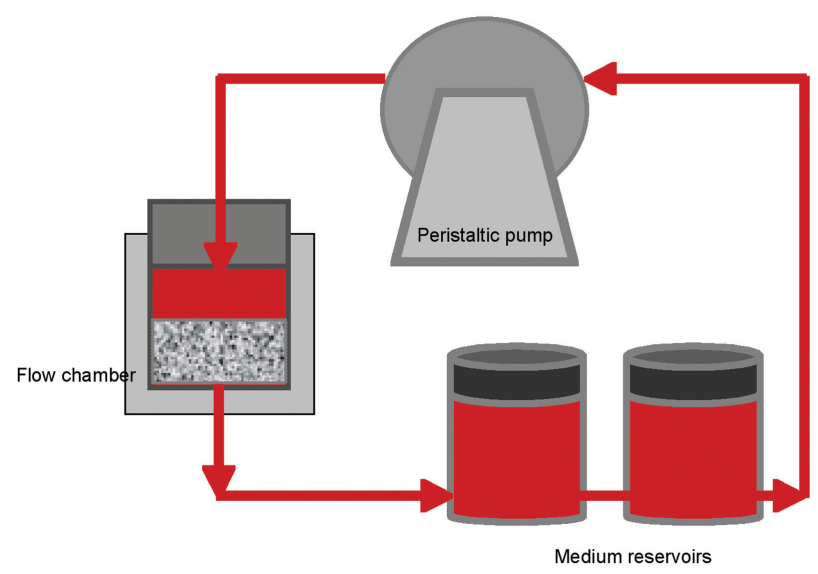

FIG. 1. Flow circuit diagram of the flow perfusion system of one of the six chambers. Adapted from Bancroft et al. ${ }^{15}$ (Color image available online at $\langle w w w . l i e b e r t p u b . c o m / t e n\rangle$. circuit diagram of the flow perfusion system of one of the six chambers.

At the end of each culture period, 4 of the 6 cell-scaffold constructs from each group were rinsed with phosphate-buffered saline (PBS) and stored at $-70^{\circ} \mathrm{C}$ in 10 $\mathrm{mL}$ tubes containing $1.4 \mathrm{~mL}$ of distilled, deionized water for DNA, calcium, and alkaline phosphatase (ALP) analyses. Before performing the assays, the samples were thawed at room temperature and then sonicated for about $15 \mathrm{~min}$. Simultaneously, at each time point, two scaffolds from each group were retrieved and fixed in a solution of $2.5 \%$ gluteraldehyde for scanning electron microscopy (SEM), Fourier transformed infrared spectroscopy with attenuated total reflectance (FTIR-ATR), and thin-film $\mathrm{X}$-ray diffraction (TF-XRD).

\section{Characterization of cultured scaffolds}

Cellularity of scaffolds. The DNA content of each scaffold was measured using a PicoGreen DNA Quantification Kit (Molecular Probes, Eugene, OR). A description of the assay can be found elsewhere. ${ }^{16}$ The cellularity of each scaffold was then calculated by correlation with the DNA of a known amount of marrow stromal cells.

Alkaline phosphatase activity. The alkaline phosphatase (ALP) activity was measured using a colorimetric endpoint assay, which measures the conversion of p-nitrophenyl phosphate to p-nitrophenol by the enzyme, alkaline phosphatase ${ }^{16}$ (Sigma Diagnostic Kit \#104).

Calcium content of scaffolds. Cell-scaffold constructs were incubated overnight in $1 \mathrm{~N}$ acetic acid to dissolve the deposited calcium. The calcium content was then measured using a colorimetric endpoint assay, which measures the amount of calcium-cresolphthalein complexone formed when the latter binds to free calcium in an alkaline solution (Sigma Diagnostic Kit \#587). The amount of deposited calcium was expressed as milligrams of $\mathrm{Ca}^{2+}$ equivalents per scaffold. ${ }^{16}$

Scanning electron microscopy. SEM analysis was performed to study the distribution and morphology/structure of the cells and matrix on the cell-scaffold constructs. For this purpose, the samples were fixed in a solution of $2.5 \%$ glutaraldehyde (in PBS), dehydrated in a series of graded ethanol solutions, dried with tetramethylsilane, and sputter coated with gold (Jeol JFC 1100). Samples were then observed using a scanning electron microscope (Leica Cambridge S360).

Fourier transformed infrared spectroscopy with attenuated total reflectance. The composition of the cell-scaffold constructs cultured for 15 days in the perfusion biore- 
A

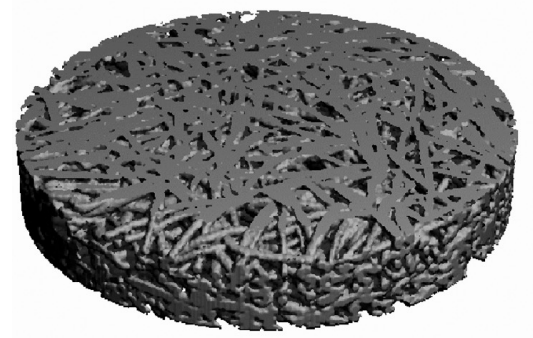

B

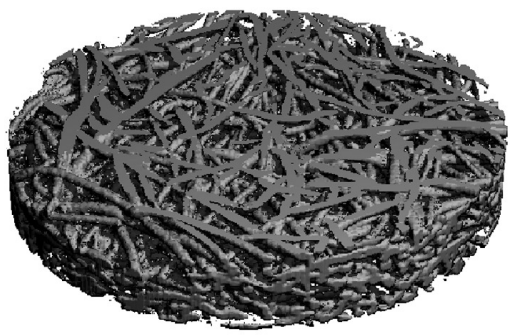

FIG. 2. Representative $\mu$ CT scans of (A) $50 \%$ porous SPCL-based scaffold and (B) $75 \%$ porous SPCL-based scaffold.

actor was analyzed by FTIR-ATR. The samples were fixed in a solution of $2.5 \%$ gluteraldehyde and dried at room temperature. All spectra were recorded using at least 64 scans and $2 \mathrm{~cm}^{-1}$ resolution in a FTIR spectrophotometer (1600 Series, Perkin-Elmer, Boston, MA) with a single reflection ATR system (MKII Golden Gate, Specac, Orpington, United Kingdom). In order to exclude any possible contributions to mineralization from the culture medium, these results were compared to cell-free scaffolds, which were exposed to the same conditions as the statically cultured constructs before being analyzed.

Thin-film X-ray diffraction. Thin-film X-ray diffraction (TF-XRD, Philips X'Pert MPD, Eindhoven, The Netherlands) was used to identify any mineral phase present in cell-scaffold constructs cultured in the perfusion bioreactor for 15 days. As for the FTIR-ATR analysis, the samples were dried at room temperature after being fixed in a solution of $2.5 \%$ gluteraldehyde. The data collection was performed by the $2 \theta$ scan method with $1^{\circ}$ incident beam angle using $\mathrm{CuK} \alpha \mathrm{X}$-ray line and a scan speed of $0.05^{\circ} / \mathrm{min}$ in $2 \theta$. Again, the results were compared to cell-free scaffolds, which were kept in the same conditions and submitted to the same treatment as samples resulting from static cultures.

\section{Statistical analysis}

Results from DNA, ALP, and calcium assays are presented as means \pm standard deviation for $n=4$. Multiple pair-wise comparisons were performed using the Tukey-Kramer method with a significance level of $95 \%$.

\section{RESULTS AND DISCUSSION}

\section{Characterization of scaffold porosity}

The two types of SPCL scaffolds produced for this study possessed a typical fiber-mesh structure (Fig. 2), with porosities of $50.5 \pm 1.9$ (Fig. $2 \mathrm{~A}$ ) and $74.5 \pm 1.2$ (Fig. 2B), respectively, as determined by $\mu \mathrm{CT}$ analyses. In this paper, the two scaffolds are referred to as $50 \%$ and $75 \%$ scaffolds, respectively.

\section{DNA analysis}

The number of cells per scaffold (calculated from DNA measurements) at the end of each culture period studied is represented in Figure 3. Initial cell attachment was $85-90 \%$, resulting in between $4.2 \times 10^{5}$ and $4.5 \times 10^{5}$ cells at day 0 (data not shown). For both scaffolds (of different porosities) and for both culture conditions, it is possible to distinguish a first period of active proliferation, correspondent to the first week of culture. A period of decreased proliferation, reflected by the decline in DNA synthesis, was then observed and is typically associated with matrix maturation and late-stage differentiation of osteoblastic cells. ${ }^{1}$ This behavior has been previously observed when marrow stromal cells were seeded

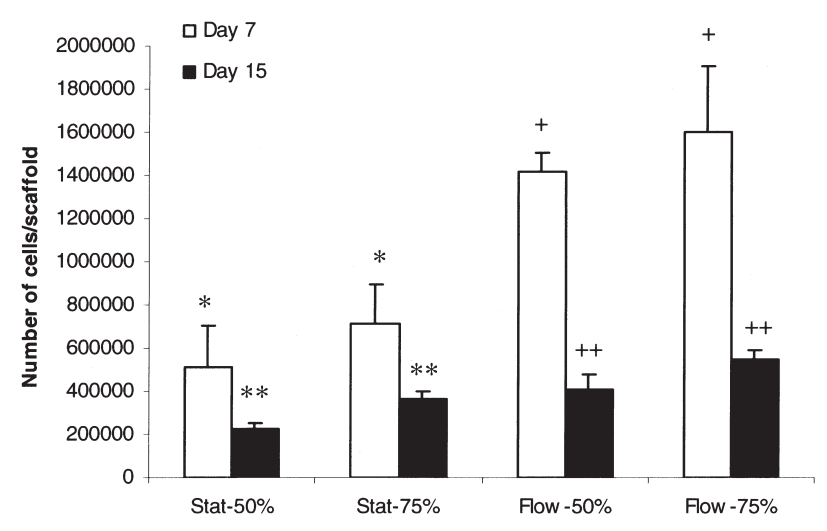

FIG. 3. Number of cells on SPCL fiber meshes-75\% porous and $50 \%$ porous-after 7 and 15 days of culture under static and flow perfusion cultures. Error bars represent means \pm standard deviation for $n=4$. Results assigned with different symbols are statistically different $(p<0.05)$, indicating: *the higher cellularity in $75 \%$ porous scaffolds as compared to $50 \%$ in static cultures after 7 days of culturing; **the higher cellularity in $75 \%$ porous scaffolds as compared to $50 \%$ in static cultures after 15 days of culturing; + the higher cellularity in $75 \%$ porous scaffolds as compared to $50 \%$ in bioreactor cultures after 7 days of culturing; ++ the higher cellularity in $75 \%$ porous scaffolds as compared to $50 \%$ in bioreactor cultures after 15 days of culturing. The cellularity is also significantly higher in the bioreactor cultures, as compared to static cultures on both scaffolds, but this is not presented in order to simplify the data displayed. 
into both starch-based scaffolds ${ }^{19}$ and titanium fiber meshes ${ }^{16,22}$ and subsequently cultured under similar conditions.

The cellularity of SPCL scaffolds with $75 \%$ porosity after 7 and 15 days of culture was significantly higher $(p<0.05)$ than that of scaffolds with $50 \%$ porosity cultured under the same conditions (static or flow perfusion conditions) for the same period of culture. These results suggest that higher porosity provides more space for cell proliferation but may also enhance diffusion of nutrients and facilitate metabolic waste removal that enables cell survival within the scaffolds.

Both $50 \%$ and $75 \%$ scaffolds cultured under flow perfusion show significantly higher $(p<0.05)$ cellularity than the same type of scaffolds cultured under static conditions, at both time points. In a previous study ${ }^{19}$ in which the flow rate during perfusion culture was lower, histology demonstrated that flow perfusion enabled a better distribution of cells and matrix within the constructs compared to static culture due to the increased mass transfer resulting from the convective fluid flow provided by the perfusion bioreactor. The higher volume of media (and consequently of nutrients and stimulating factors) available for the constructs cultured in the perfusion bioreactor, as compared to constructs kept under static conditions, may also influence the proliferation and differentiation of the cells. The results of the present study agree with these results. In addition, the comparison of the cellularity of $75 \%$ porous scaffolds with the previous results ${ }^{19}$ obtained for the same type of scaffold demon- strates that increasing flow rate leads to enhanced cell proliferation, as was previously shown for titanium fiber meshes cultured under similar conditions. ${ }^{16}$

\section{Scanning electron microscopy}

SEM images obtained from the top surface (where cells were seeded) of $50 \%$ and $75 \%$ porous scaffolds after 15 days of culture in the flow perfusion bioreactor (Fig. 4) show the presence of a dense cell matrix filling the constructs. The bottom surface of the $75 \%$ porous scaffolds was also completely covered with a dense matrix, whereas the bottom surface of the $50 \%$ porous scaffolds was not completely covered. Thus, the lower porosity appears to hinder the penetration and distribution of cells throughout the entire construct. This observation is in agreement with the results obtained from DNA measurements, which indicate a higher cell number present in the $75 \%$ porous meshes. Additionally, in the higher porosity scaffolds, the flow perfusion induced de novo tissue modeling with the formation of pore-like structures within the deposited extracellular matrix. The same is not observed in the $50 \%$ porous scaffolds. Thus, scaffold porosity appears to affect both the amount of cells and matrix formed within the construct, as well as the structure of this newly formed tissue.

\section{Alkaline phosphatase analysis}

Figure 5 depicts the normalized alkaline phosphatase activity of marrow stromal cells seeded onto scaffolds
A1

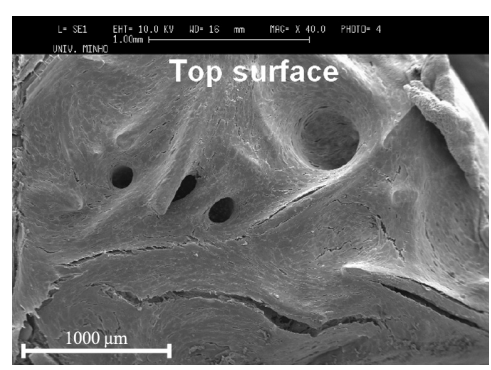

A2

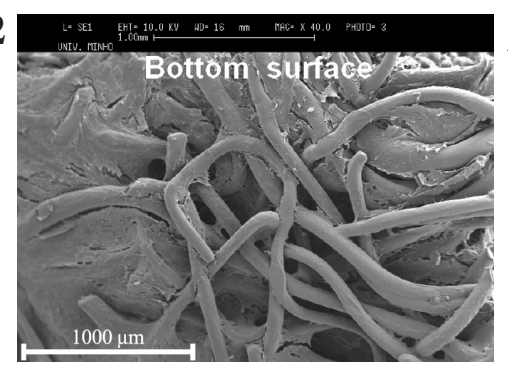

A3

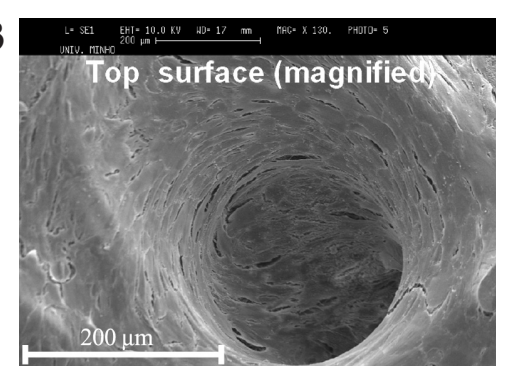

B2

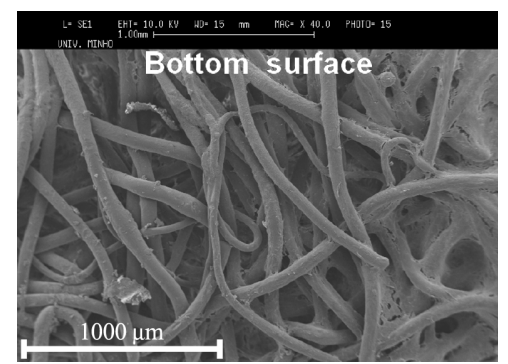

B1

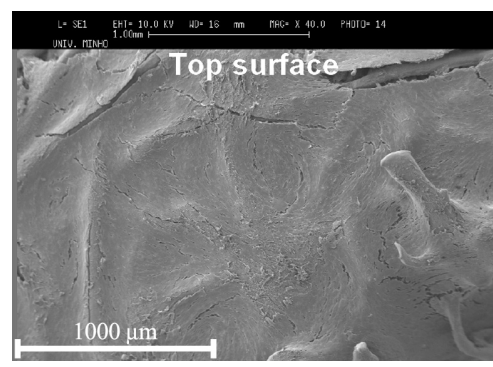

FIG. 4. SEM images depicting the surface of SPCL fiber meshes with (A) $75 \%$ porosity and (B) $50 \%$ porosity cultured for 15 days in the flow perfusion bioreactor. (A1 and B1) Top surface (surface where cells were seeded) and (A2 and B2) bottom surface (opposite to the surface where cells were seeded) of samples (bar is $1000 \mu \mathrm{m}$ ); (A3) magnification of A1, showing in detail the pore-like structure formed in $75 \%$ scaffolds (bar is $200 \mu \mathrm{m}$ ). 


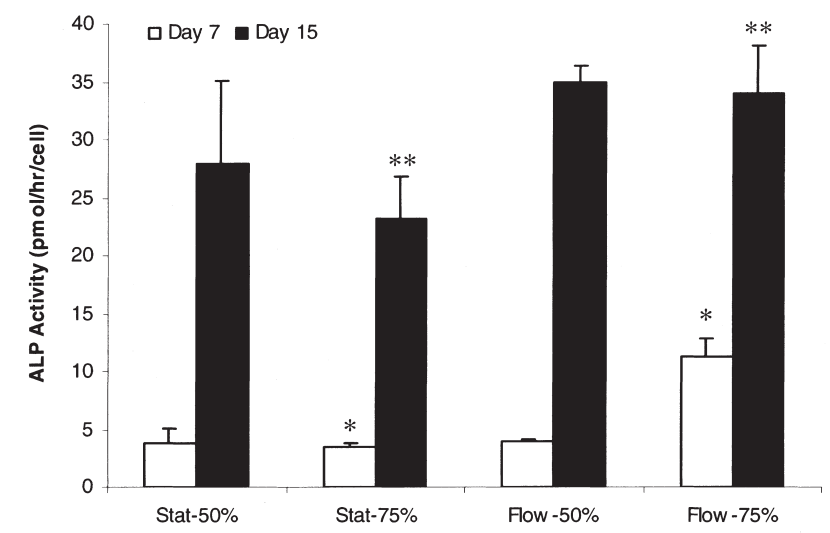

FIG. 5. Normalized ALP activity of marrow stromal cells after 7 and 15 days of culture on SPCL fiber meshes with $75 \%$ and $50 \%$ porosity. Error bars represent means \pm standard deviation for $n=4$. Statistically different results $(p<0.05)$ indicate: *the significantly higher level of ALP activity observed for $75 \%$ scaffolds cultured under flow perfusion compared to static cultures for 7 days of culture; **the significantly higher level of ALP activity observed for $75 \%$ scaffolds cultured under flow perfusion, compared to static cultures for 15 days of culture.

with different porosities after 7 and 15 days of culture under static and flow perfusion conditions. ALP is a protein associated with the bone cell phenotype, and its expression is found to significantly increase during the stage of active proliferation of osteoblastic cells. ${ }^{1}$ During matrix maturation, the extracellular matrix undergoes several modifications in composition and organization, ultimately progressing to matrix mineralization. At this point, every cell expresses alkaline phosphatase. ${ }^{1}$ In the present study, a significant increase in ALP activity from days 7 to 15 of culture was observed for all samples cultured under static and flow perfusion conditions, in agreement with the patterns described in the literature. ${ }^{1}$ In this case, the scaffold porosity did not have a strong influence on this marker of osteoblastic differentiation, as no significant difference was found between the ALP levels of 50\% and $75 \%$ porous scaffolds. However, significantly higher ALP expression was found in perfusion cultures of $75 \%$ porous scaffolds when compared to statically cultured $75 \%$ porous scaffolds at the same time point. Enhanced ALP activity in perfusion cultures has been previously observed with titanium fiber meshes. ${ }^{22}$ Since this trend was not observed with 50\% porous scaffolds, both porosity and fluid flow appear to affect the sequential development of osteogenic cells. Additionally, ALP is known to be a transient marker of osteoblastic differentiation that is downregulated as differentiation progresses. Because samples were analyzed at only two time points, other statistical differences in ALP levels between scaffold types and/or time points may have been over- looked as a result of ALP activity peaking in between time points. However, these time points do allow for adequate determination of statistical differences in endstage markers such as calcium deposition.

\section{Calcium deposition}

Figure 6 displays the calcium deposition per scaffold in $50 \%$ and $75 \%$ porous scaffolds cultured under static and flow perfusion conditions after 7 and 15 days. Mineral accumulation is a consequence of pre-osteoblastic cell progression through the proliferation and matrix maturation stages of differentiation. Calcium deposition is an essential step for the further upregulation or expression of genes responsible for the mineralization of the extracellular matrix. ${ }^{1}$ In this study, almost no calcium was measured in scaffolds by the end of the first week of both static and flow perfusion culture. However, at the end of the second week, a dramatic increase in calcium deposition was observed in scaffolds cultured in the flow perfusion bioreactor. In fact, the calcium deposited on the scaffolds cultured under flow perfusion conditions (both for 75 and $50 \%$ porous scaffolds) after 15 days of culture was significantly higher $(p<0.05)$ than calcium deposited on scaffolds cultured under static conditions. These trends are in agreement with previous results for culture of marrow stromal cells on starch-based polymer scaffolds in a flow perfusion bioreactor. ${ }^{19}$

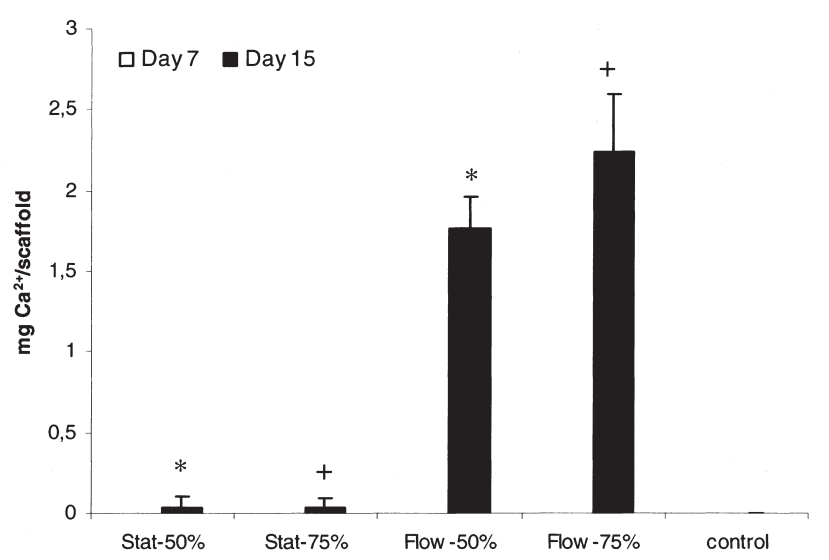

FIG. 6. Calcium deposition on SPCL fiber meshes-75\% porous and $50 \%$ porous-after 7 and 15 days of culture under static and flow perfusion conditions. Control refers to samples without cells which were "cultured" under static conditions. Error bars represent means \pm standard deviation for $n=4$. Statistically different results $(p<0.05)$ indicate: *the significantly higher calcium deposition after 15 days of culture in flow cultures as compared to static cultures of $50 \%$ porous scaffolds; + the significantly higher calcium deposition after 15 days of culture in flow cultures as compared to static cultures of $75 \%$ porous scaffolds. 
Calcium deposition was seen to increase with increasing porosity, but no significant differences between scaffold types were observed. Shear forces experienced by cells are expected to increase in fiber meshes exhibiting lower porosity. These forces can be calculated assuming a cylindrical pore model approximation for the scaffold pore morphology. ${ }^{23}$ The shear forces experienced by the cells seeded into $75 \%$ and $50 \%$ porous scaffolds were estimated to be on the order of 0.2 and $0.3 \mathrm{dyn} / \mathrm{cm}^{2}$, respectively. Previous work has shown that increasing shear forces are associated with increasing calcium deposition in titanium fiber mesh scaffolds. $^{22}$ The higher calcium deposition observed in this study for scaffolds of higher porosity may be explained by the higher number of cells and cell density registered in these scaffolds (as demonstrated by the DNA analysis), which is a very important factor in mineralization. These results further support the importance of scaffold structure and culture conditions on the osteogenic differentiation of bone marrow stromal cells.

The results obtained for the $75 \%$ porous scaffolds using a flow rate of $1 \mathrm{~mL} / \mathrm{min}$ are also significantly higher than those obtained in a previous study ${ }^{19}$ for the same scaffolds cultured at lower flow rates $(0.3 \mathrm{~mL} / \mathrm{min})$. This effect has been observed in other studies ${ }^{16,22}$ and is associated with increased mechanical stimulation induced by the increased fluid shear stresses experienced by the cells cultured under higher flow rates.

\section{FTIR-ATR}

To demonstrate the clinical feasibility of tissueengineered bone, rapid mineralization of osteoid tissue grown in vitro must be achieved to sufficiently match the intrinsic properties of autogenous bonegraft material. ${ }^{7}$ Nevertheless, the analysis of mineral deposition occurring in in vitro cultures is frequently based on von Kossa or Alizarin red staining or by calcium uptake. ${ }^{24}$ However, the results arising from these analyses can be misinterpreted, as the matrix is known to uptake calcium independently of mineral deposition. Therefore, these methods should be complemented with diffraction or spectroscopy methods. $^{24}$

In this study, FTIR-ATR spectra were obtained from 75 and $50 \%$ porous meshes cultured for 15 days in the perfusion bioreactor (Fig. 7). These spectra showed the presence of amide bands at about $1634 \mathrm{~cm}^{-1}$ (amide I) and $1525 \mathrm{~cm}^{-1}$ (amide II), which can be assigned to the protein matrix formed.

The FTIR-ATR spectra also display reflectance peaks associated with the phosphate group in carbonated apatite (group bands at $1041 \mathrm{~cm}^{-1}$ and $562 \mathrm{~cm}^{-1}$ ), clearly suggesting the presence of mineralized extracellular matrix. The spectra collected for both $50 \%$ and $75 \%$ porous scaffolds were identical.

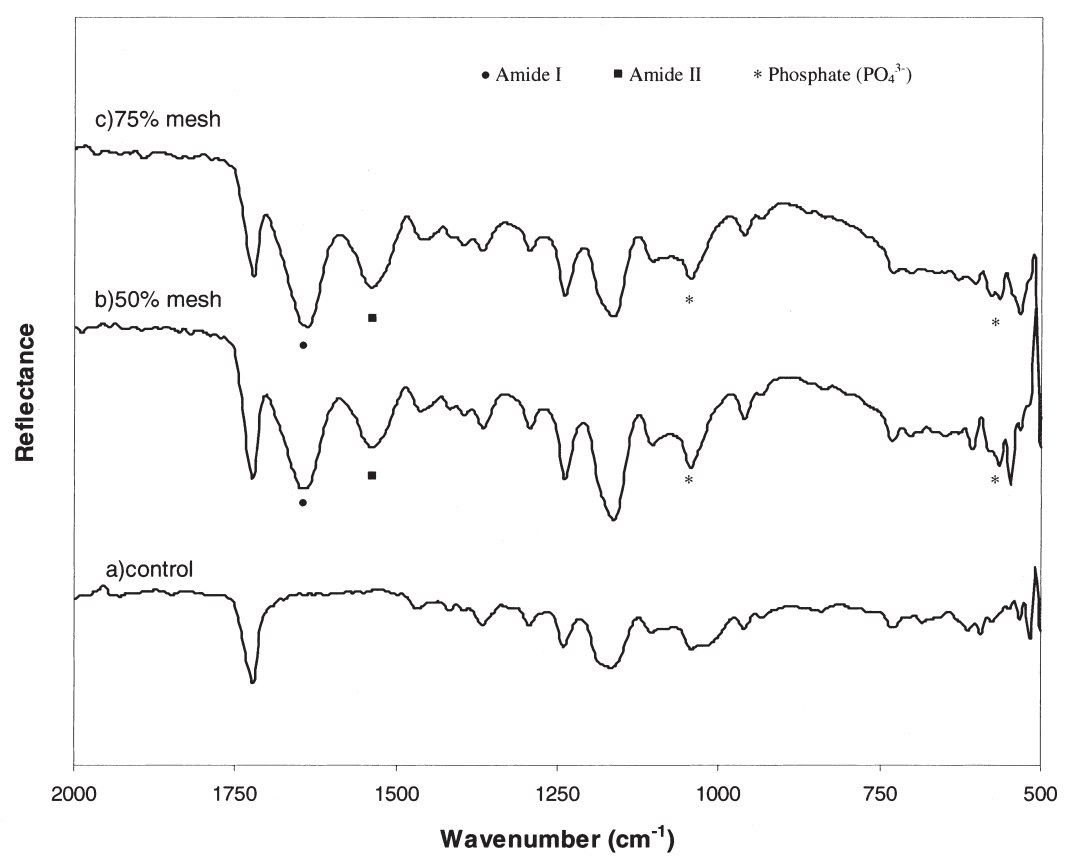

FIG. 7. FTIR-ATR spectra of (a) control, cell-free scaffold kept in the same conditions as that for static cultures and treated with the same fixative solution; (b) $50 \%$ porous scaffolds cultured in the flow perfusion bioreactor for 15 days; and (c) $75 \%$ porous scaffolds cultured in the flow perfusion bioreactor for 15 days. 


\section{$T F-X R D$}

Figure 8 shows the TF-XRD patterns of cell-scaffold constructs of different porosities after 15 days of culture in the flow perfusion bioreactor using cell-free scaffolds as controls. In these diffraction patterns, several characteristic peaks of hydroxyapatite were visible, which were confirmed by comparison to an XRD pattern of standard hydroxyapatite (JCPDS 9-432). The apatite formed appeared to be mainly amorphous, as is typically observed for minerals occurring in the presence of proteins, such as apatite in bone. ${ }^{24}$

The patterns corresponding to $50 \%$ porous scaffolds are similar to those seen for the $75 \%$ scaffolds. However, it was found that the diffraction peaks of the apatite phase exhibited lower intensity in $50 \%$ porous scaffolds, and one of the peaks, correspondent to the plane (210) as-

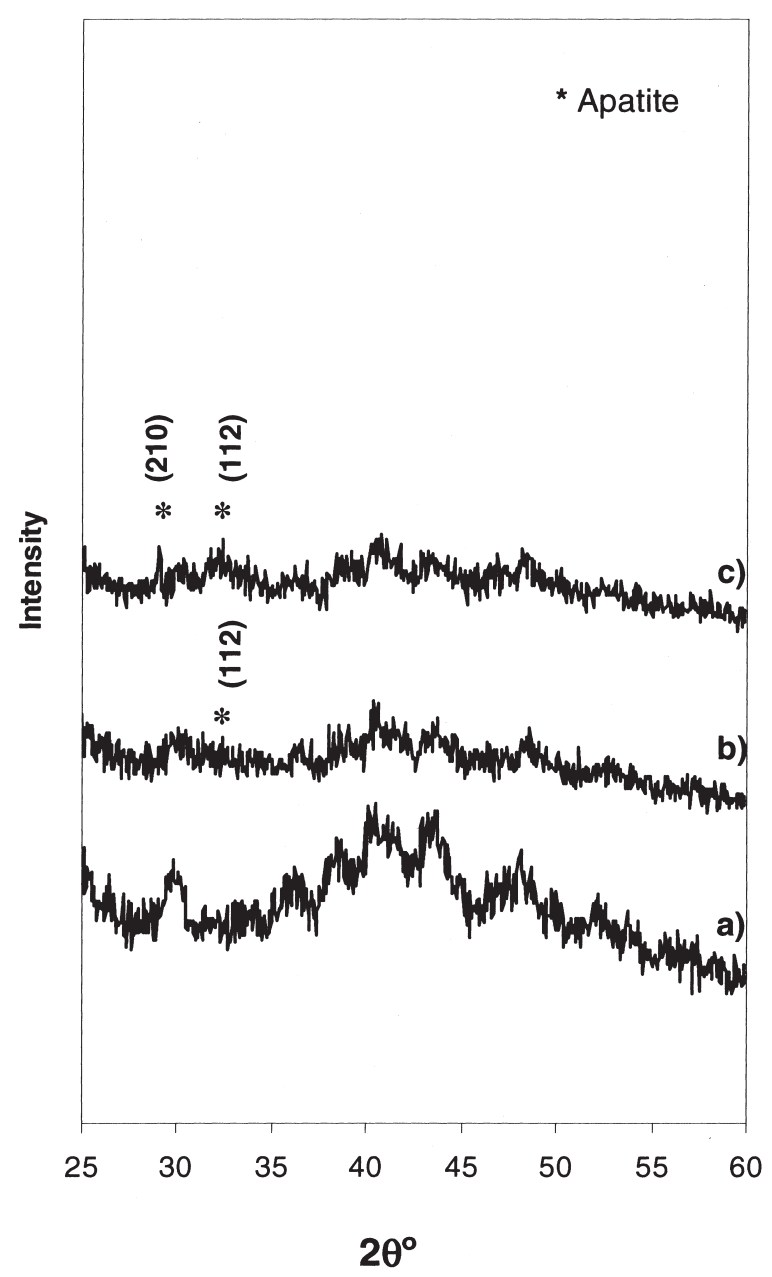

FIG. 8. TF-XRD patterns of a) Control, cell-free scaffold kept in the same conditions as that for static cultures and treated with the same fixative solution; b) $50 \%$ porous scaffolds cultured in the flow perfusion bioreactor for 15 days; and c) $75 \%$ porous scaffolds cultured in the flow perfusion bioreactor for 15 days. signed in the patterns of the $75 \%$ porous scaffold, is not evidenced in the patterns correspondent to $50 \%$ porous scaffolds. This might be a further indication of the influence of porosity in the development of the mineralized extracellular matrix by marrow stromal cells cultured under flow perfusion conditions. These results, together with the FTIR-ATR spectra, suggest that the mineral formed by marrow stromal cells seeded in SPCL fiber meshes and cultured under flow perfusion conditions is a carbonated apatite mineral similar to the major mineral component of bone. ${ }^{26}$

\section{CONCLUSIONS}

This study shows that biodegradable starch-based fiber mesh scaffolds in conjunction with fluid flow bioreactor culture enable the creation of culture environments with minimal diffusional constraints and the appropriate mechanical stimulation for marrow stromal cell differentiation toward production of a mineralized bone-like extracellular matrix. This mineralized matrix contained carbonated apatite mineral similar to the major mineral component of bone.

This study demonstrates that increased scaffold porosity significantly enhances the proliferation of marrow stromal cells cultured under static and flow perfusion conditions and influences the sequential development of the seeded cells. Furthermore, flow perfusion was seen to induce de novo tissue modeling with the formation of pore-like structures in the scaffolds of higher porosity. These results demonstrate that this structural aspect of scaffolds, in combination with the culture environment, influences the structure and possibly the functionality of bone-like tissue substitutes formed in vitro.

The culturing of highly porous starch-based fiber mesh scaffolds seeded with marrow stromal cells under flow perfusion conditions may allow for the development of adequate in vitro engineered substitutes for the repair of bone tissue.

\section{ACKNOWLEDGMENTS}

M.E. Gomes acknowledges the Portuguese Foundation for Science and Technology (FCT), Grant SFRH/ BD/4704/2001. The authors would like to thank Dr. Francesco Degli-Innocenti from Novamont, Italy, for supplying the starch-based polymers, Jeremy Lemoine at Rice University for the $\mu \mathrm{CT}$ imaging work, and Isabel Leonor for help on the FTIR-ATR and TF-XRD analyses. The authors also acknowledge financial support from the National Institutes of Health (R01-AR42639) (AGM) and from FCT through funds from the POCTI and/or FEDER programs. 


\section{REFERENCES}

1. Lian, J.B., and Stein, G.S. Concepts of osteoblast growth and differentiation: basis for modulation of bone cell development and tissue formation. Crit. Rev. Oral. Biol. Med. 3, 269, 1992.

2. Li, Y., Ma, T., Yang, S.T., and Kniss, D.A. Thermal compression and characterization of three-dimensional nonwoven PET matrices as tissue engineering scaffolds. Biomaterials 22, 609, 2001.

3. Wang, Y., Uemura, T., Dong, J., Kojima, H., Tanaka, J., and Tateishi, T. Application of perfusion culture system improves in vitro and in vivo osteogenesis of bone marrow-derived osteoblastic cells in porous ceramic materials. Tissue Eng. 9, 1205, 2003.

4. Sikavitsas, V.I., Temenoff, J.S., and Mikos, A.G. Biomaterials and bone mechanotransduction. Biomaterials 22, 2581, 2001.

5. Tera, H., Hannouche, D., Ochoa, E., Yamano, Y., and Vacanti, J.P. In vitro engineering of bone using a rotational oxygen-permeable bioreactor system. Mat. Sci. Eng. CBio. S. 20, 3, 2002.

6. Calvert, J.W., Marra, K.G., Cook, L., Kumta, P.N., DiMilla, P.A., and Weiss, L.E. Characterization of osteoblast-like behavior of cultured bone marrow stromal cells on various polymer surfaces. J. Biomed. Mater. Res. 52, $279,2000$.

7. Botchwey, E.A., Pollack, S.R., Levine, E.M., and Laurencin, C.T. Bone tissue engineering in a rotating bioreactor using a microcarrier matrix system. J. Biomed. Mater. Res. 55, 242, 2001.

8. Leong, K., Cheah, C., and Chua, C. Solid free fabrication of three-dimensional scaffolds for engineering replacement tissue and organs. Biomaterials 24, 2363, 2003.

9. Ma, P.X., and Choi, J.W. Biodegradable polymer scaffolds with well-defined interconnected spherical pore network. Tissue Eng. 7, 23, 2001.

10. Murphy, W.L., Dennis, R.G., Kileny, J.L., and Mooney, D.J. Salt fusion: an approach to improve pore interconnectivity within tissue engineering scaffolds. Tissue Eng. 8, 43, 2002.

11. Shea, L.D., Wang, D., Franceschi, R.T., and Mooney, D.J. Engineered bone development from a pre-osteoblast cell line on three-dimensional scaffolds. Tissue Eng. 6, 605, 2000.

12. Wake, M.C., Patrick, C.W., and Mikos, A.G. Pore morphology affects on the fibrovascular tissue growth in porous polymers substrates. Cell Transplant. 3, 339, 1994.

13. Butler, D.L., Goldstein, S.A., and Guilak, F. Functional tissue engineering: the role of biomechanics. J. Biomech. Eng. 122, 570, 2000.

14. Cowin, S.C. On mechanosensation in bone under microgravity. Bone 22, 119, 1998.

15. Bancroft, G.N., Sikavitsas, V.I., and Mikos, A.G. Design of a flow perfusion bioreactor system for bone tissue-engineering applications. Tissue Eng. 9, 549, 2003.
16. Bancroft, G.N., Sikavitsas, V.I., van den Dolder, J., Sheffield, T.L., Ambrose, C.G., Jansen, J.A., and Mikos, A.G. Fluid flow increases mineralized matrix deposition in 3D perfusion culture of marrow stromal osteoblasts in a dose-dependent manner. Proceed. Natl. Acad. Sci. USA 99, 12600, 2002.

17. Temenoff, J.S., and Mikos, A,G. Tissue engineering for regeneration of articular cartilage. Biomaterials 21, 431, 2000.

18. Freed, L.E., and Vunjak-Novakovic, G. Tissue engineering bioreactors. In: Lanza R., Langer R., and Vacanti J.P., eds., Principles of Tissue Engineering. San Diego: Academic Press, 2000, pp.143-156.

19. Gomes, M.E., Sikavitsas, V.I., Behravesh, E., Reis, R.L., and Mikos, A.G. Effect of flow perfusion on the osteogenic differentiation of bone marrow stromal cells cultured on starch-based three-dimensional scaffolds. J. Biomed. Mater. Res. 67A, 87, 2003.

20. Behravesh, E., Timmer, M.D., Lemoine, J.J., Liebschner, M.A.K., and Mikos, A.G. Evaluation of the in vitro degradation of macroporous hydrogels using gravimetry, confined compression testing, and microcomputed tomography. Biomacromolecules 3, 1263, 2002.

21. Maniatopoulos, C., Sodek, J., and Melcher, A.H. Bone formation in vitro by stromal cells obtained from bone marrow of young adult rats. Cell Tissue Res. 254, 317, 1988.

22. Sikavitsas, V.I., Bancroft, G.N., Holtorf, H.L., Jansen, J.A., and Mikos, A.G. Mineralized matrix deposition by marrow stromal osteoblasts in 3D perfusion culture increases with increasing fluid shear forces. Proceed. Natl. Acad. Sci. USA 100, 14683, 2003.

23. Goldstein, A.S., Juarez, T.M., Helmke, C.D., Gustin, M.C., and Mikos, A.G. Effect of convection on osteoblastic cell growth and function in biodegradable polymer foam scaffolds. Biomaterials 22, 1279, 2001.

24. Boskey, A.L. Biomineralization: conflicts, challenges, and opportunities. J. Cell Biochem. 30-31 suppl., 83, 1998.

25. Wen, H.B., de Wijn, J.R., van Blitterswijk, C.A., and de Groot, K. Incorporation of bovine serum albumin in calcium phosphate coating on titanium. J. Biomed. Mater. Res. 46, 245, 1999.

26. Mann, S. Biomineralization, Principles and Concepts in Bioinorganic Materials Chemistry. Oxford: Oxford University Press, 2001.

\footnotetext{
Address reprint requests to: Antonios G. Mikos, Ph.D.

Department of Bioengineering Rice University P.O. Box 1892 MS 142 Houston, TX 77251-1892

E-mail: mikos@rice.edu
} 